\title{
Smart Grid Information Management System Relying on MAS Technology and Complex Scientific Management Thinking
}

\author{
Jing Tong (iD) \\ School of Electrical and Electronic Engineering, Hubei University of Technology, Wuhan 430000, China \\ Correspondence should be addressed to Jing Tong; 20050046@hbut.edu.cn
}

Received 25 November 2021; Accepted 27 January 2022; Published 7 March 2022

Academic Editor: Daqing Gong

Copyright (๑) 2022 Jing Tong. This is an open access article distributed under the Creative Commons Attribution License, which permits unrestricted use, distribution, and reproduction in any medium, provided the original work is properly cited.

\begin{abstract}
With the continuous social and economic development, more and more attention has been paid to the safety and efficiency of the power grid. In this paper, the importance and technical support for the informatization and intelligent implementation of the smart grid are explored based on the complex scientific management thinking combined with MAS technology, with the smart grid as the breakthrough point. Subsequently, the framework structure of the smart grid information management system based on multiagent technology is put forward, focusing on expounding the design and functions of agents at the equipment level, subsystem level, and total system level in the system. The system functions are verified and analyzed through the test examples. The practice has proved that the smart grid information management system designed in this paper can provide technical support for smart grid informatization and intelligent implementation.
\end{abstract}

\section{Introduction}

With the continuous social and economic development, more and more attention has been paid to the safety and efficiency of the power grid [1-4]. Informatization and intelligence are a game changer to the power grid industry, and a novel type of information system that can cover the power grid comprehensively is the basis for implementing a smart power grid. With the introduction and application of sensor networks, the operating status of power grid equipment, the peripheral working environment, and other pieces of information can be monitored, which has provided support for the implementation of informatization in the smart power grid [5-8]. Although the energy management system (EMS) has been extensively applied in power grid dispatching management, it still requires the direct involvement of dispatchers and distributed independent computation. Then dispatchers make decisions and process information based on experience, which can reduce the real-time performance and accuracy of dispatching operations [9-12]. As the scale of power grids is continuously expanded, with the deepening of the power market reform and the advent of the comprehensive construction of smart power grids, the operation mode and structural adjustment of the power system have become increasingly complicated, which has put forward more rigorous requirements on the psychological quality and operating experience of dispatchers. Upon failure of the power grid, massive alarm information will be displayed to the dispatcher right away, and it will be impossible for the dispatcher to analyze and process the information in a short time. It will also be highly challenging to make scientific and accurate processing decisions $[13,14]$. Hence, the operation of the power grid requires a dispatch automation system with more comprehensive functions, more intelligent information processing, and more scientific decision-making urgently to improve the economy and safety in the operation of the power grid [15].

Smart grid information management system (SGIMS) puts forward higher requirements, which requires it to evolve into a complex adaptive system (CAS) with intelligent characteristics, with good flexibility, scalability, and adaptability. It can not only effectively realize the data collection, prediction, and intelligent functions such as planning, selforganization, self-control, and auxiliary decision-making but also complete the optimal production, operation, and transaction of power in the complex information 
environment. It includes regular inspection and testing of the operation of key facilities in terms of regulatory control and data collection and the realization of scheduling and other auxiliary service functions based on a cost-effective mechanism. However, China's previous power information management mainly used EMS and other types of application software, which does not have the ability of intelligent information decision-making, so it is difficult to meet the complex information management requirements of smart grid. Vigorously developing information technology facilities matching the goal of smart grid has become an important application research field. At present, MAS technology is mostly used in the field of distributed artificial intelligence in complex system science and is also widely used in resource management, risk management, and network information system management. Similarly, MAS technology can also provide a feasible way for the function realization of smart grid information management system. At present, this technology and method have been preliminarily applied and studied in the fields of power line transmission application scenario of smart grid, structural design of distributed high-security control system, power dispatching, and system relay protection (such as wide-area backup protection, coordinated protection, online verification, and early warning system).

In this paper, the design of the smart grid information management system is studied based on the complex scientific management thinking combined with the MAS technology, and the corresponding information management system is established. Finally, the proposed system is verified and analyzed based on the practical data to explore the management analysis of the smart grid industry.

\section{Related Technologies and Methods}

2.1. MAS Technology. The electric power system is a realtime dynamic system composed of five essential links: power generation, transmission, transformation, power distribution, and power consumption. The prerequisites to ensure the stable operation of the electric power system are as follows. The system is required to meet two basic equations, that is, the equality constraint and the inequality constraint. Firstly, from the process of power reproduction, it can be divided into intelligent modules of power generation, transmission, transformation, distribution, and power consumption. These modules centralize the power demand information of users. Among them, the power generation information agent can support different power access, including a large amount of clean power generation information such as distributed power generation. The generation, transmission, transformation, and distribution information agent centralizes the power supply side information. The power information agent centralizes the power demand side information and can provide information services for power market transactions under the demand side response. Dispatching information agent centralizes a large amount of power transaction information to realize power dispatching based on economic and environmental efficiency. The equation constraint condition for the electric power system means that the active power and reactive power of the system shall maintain a dynamic balance in real time, which can be expressed by equations (1) and (2):

$$
\left\{\begin{array}{l}
\sum_{i=1}^{i=n} P_{G i}=\sum_{j=1}^{j=n} P_{L j}+\sum_{k=1}^{k=n} P_{s k}, \\
\sum_{i=1}^{i=n} Q_{G i}=\sum_{j=1}^{j=n} Q_{L j}+\sum_{k=1}^{k=n} Q_{s} .
\end{array}\right.
$$

$P_{G i}$ and $Q_{G i}$ stand for the active power and reactive power generated by the $i$ th power supply in the system; $n$ stands for the number of power nodes; $P$ and $Q$ represent the active power and reactive power of power in the system; $P_{s k}$ and $Q_{s k}$ stand for the active and reactive power loss of the $K$ th power transmission and transformation equipment in the system; $m$ stands for the number of loads in the system.

$$
\left\{\begin{array}{l}
U_{i, \min } \leq U_{i} \leq U_{i, \max }, \\
f_{\min } \leq f \leq f_{\max }, \\
P_{G i, \min } \leq P_{G i} \leq P_{G i, \max }, \\
Q_{G i, \min } \leq Q_{G i} \leq Q_{G i, \max } .
\end{array}\right.
$$

$U_{i, \min }, U_{i}$, and $U_{i, \max }$ stand for the voltage and upper and lower limits of the $i$-th power supply of the bus, respectively; $f_{\min }, f$, and $f_{\max }$ stand for the frequency and upper and lower limits of the system, respectively; $P_{G i \text {,min }}, P_{G i}$, and $P_{G i \text { max }}$ stand for the upper and lower limits of the active power output of the $i$-th generator, respectively; $Q_{G i \text {,min }}, Q_{G i}$, and $Q_{G i \text {,max }}$ stand for the upper and lower limits of the reactive power output of the $i$-th generator, respectively.

The power grid dispatching system is a real-time dynamic system with a huge amount of information and a large scale. For the safe, economical, and high-quality operation of the power grid, it is required to deal with the minimization of system network loss, the stability of voltage quality and frequency quality at the same time, and other issues, which can be regarded as an ultrahigh-dimensional, nonlinear, and differential equation-inequality constraint conditional variation problem. Its mathematical model is shown in equations (3) and (4):

$$
\begin{aligned}
& \min J(x, y)=\int_{0}^{\infty} \sum_{i=1}^{n} q_{i} F(x, y), \\
& \left\{\begin{array}{l}
\text { s.t. } x=f(x, u) \\
0=\Phi(x, y) \\
\zeta<\Delta f<\xi \\
\alpha<\Delta V<\beta
\end{array}\right.
\end{aligned}
$$

where $x \in R^{n}$ and $X \in R^{n}$ (in which $R$ is a real number) stand for the state vector and output vector of the power system, $u \in R^{r}$ stands for the control vector; $f(x, u)$ is the firstorder state equation; $\Delta f$ and $\Delta V$ stand for the voltage and frequency dynamic deviation of the node, respectively. As the nodes in the power grid are huge and complex, it is impossible to solve the aforesaid problem in a short time (10 s), which is also the reason why the dispatch automation 
system can only be used for analysis but not for global solution control at present.

Interaction with the environment is a prerequisite for the technical work of the agent. Subsequently, the environmental information received is processed. The working process is shown in Figure 1.

The multiagent system, referred to as MAS for short, is a set of multiple agents. The individual agents in the set collaborate with each other to implement a specific function. Upon implementation of the information relationship, control relationship, and solution mode among the internal agents of MAS, there are some differences in the organizational structure. When the organizational structures are different, the information storage, sharing, and communication methods between agents can be determined. When the organizational structures are the same, the agents have the same information communication mechanism. Hence, the organizational structures of MAS can be divided into three types based on various management modes and service mechanisms: centralized, distributed, and hybrid. Their structures are shown in Figure 2.

The operation of the system requires the establishment of a common platform, while the SACDA, EMS, and DMS applied in the dispatch automation system at present work independently in different environments. It is highly challenging to coordinate the relationship between the three. Hence, it is necessary to establish a system platform with a layered and distributed architecture. The functional modules of the system should be independent of each other, and coordinated operation should be maintained, which cannot be met based on the traditional object-oriented technology. The MAS technology is applied to the field of the power dispatch automation system, and the latest cutting-edge technologies are integrated, such as computer technology, information processing technology, and communication technology. The EMS is upgraded to an agent-based intelligent dispatch control system, and an intelligent dispatch management model is established, which has provided an effective solution to the complex and changeable issues in the power system.

\subsection{Design of the Smart Grid Information Management System} Based on Complex Scientific Management Thinking and MAS Technology. The multiagent system is composed of a limited number of single agents based on a certain topological structure. The single agent of the smart grid information management system herein mainly refers to the subagents such as transmission lines, transformers, and circuit breakers at the equipment level, the subagents at each voltage level in the subsystem, and the subagents of the overall system. The aforesaid subagents acquire real-time data information of the power grid through the monitoring system and other agents and analyze and evaluate the current state of themselves and the environment.

The results are shared with other agents and then combined with the state of the power grid to develop the control strategy in the next step, cooperate with other agents to accomplish the control task, finally organize the analysis

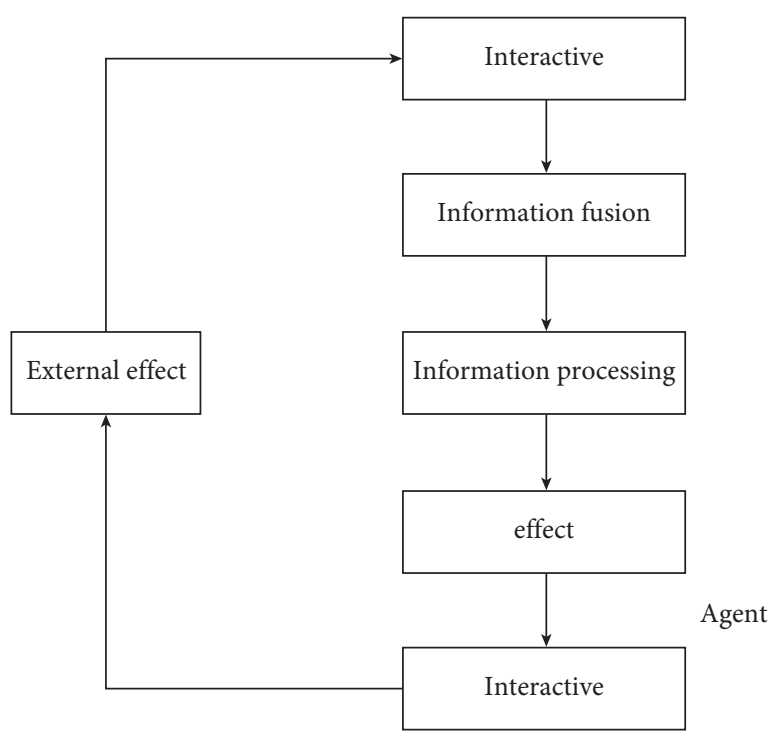

FIgURE 1: Working process of the agent.

results and control strategy into a log and report to the superior agent, and wait for further instructions from the superior agent.

The design of a single agent is shown in Figure 3.

The smart grid-oriented information management system established in this paper is mainly composed of three types of agents: agent at the total system level, agent at the subsystem (substation) level, and agent at the equipment level. The agents have the structure as shown in Figure 3. In addition, their coordination module, analysis module, communication management module, and data management module are essentially similar. The difference only lies in the algorithm implementation. In addition, the agents of the state monitoring module and the execution module are different at all levels, and agents at various levels have different information sources and control objects.

The smart power grid information management system based on the multiagent system (MAS) is composed of subagents, as shown in Figure 3. The multiagent system (MAS) is composed of multiple loosely coupled, coarsegrained agents. While taking the advantages of the singleagent system into consideration, the critical point is that the function-independent agents can accomplish complex distributed tasks and solve the problems through collaboration with each other.

In this paper, the star network is adopted as the communication mode to meet the demand of the rapid and accurate interaction of a large amount of information in the smart power grid. The communication module of the upperlevel agent in each subagent group also takes the coordination of the communication between the subagents into consideration and functions as the central server of each subagent and the transfer station for information flow. The network address, geographic location, electrical properties, and other pieces of information of each subagent are stored in the database. The system design is shown in Figure 4.

In Figure 4, $A$ stands for the total system agent (agent), $A_{i}$ stands for the subsystem (substation) and equipment 

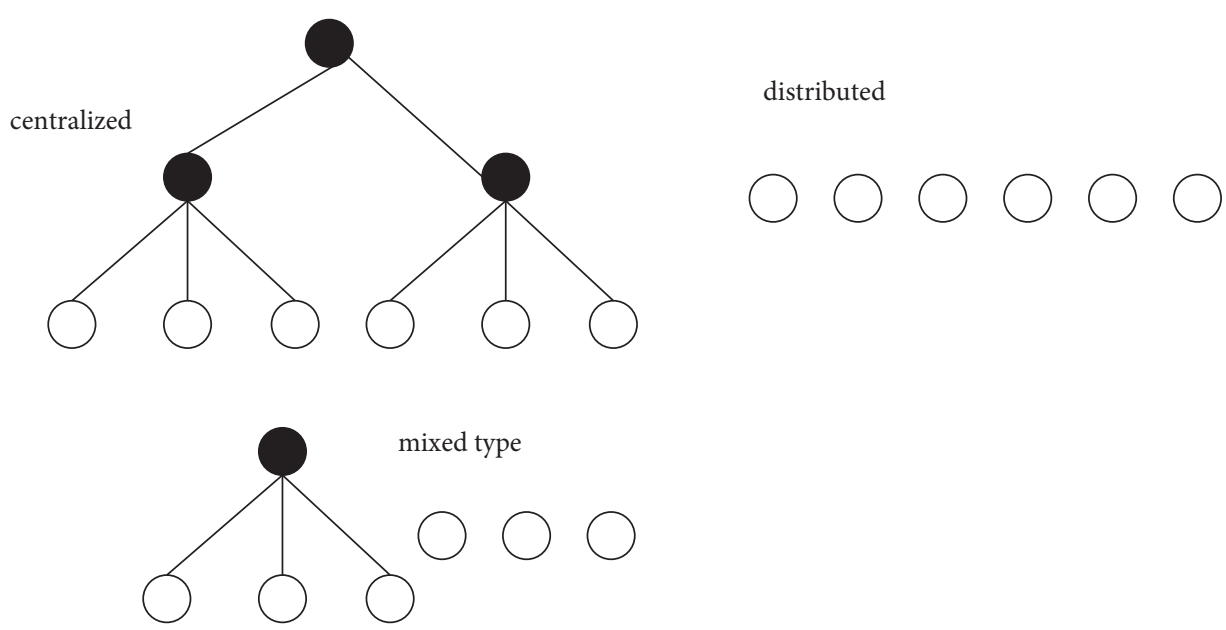

FIgURE 2: Organizational form of the MAS.

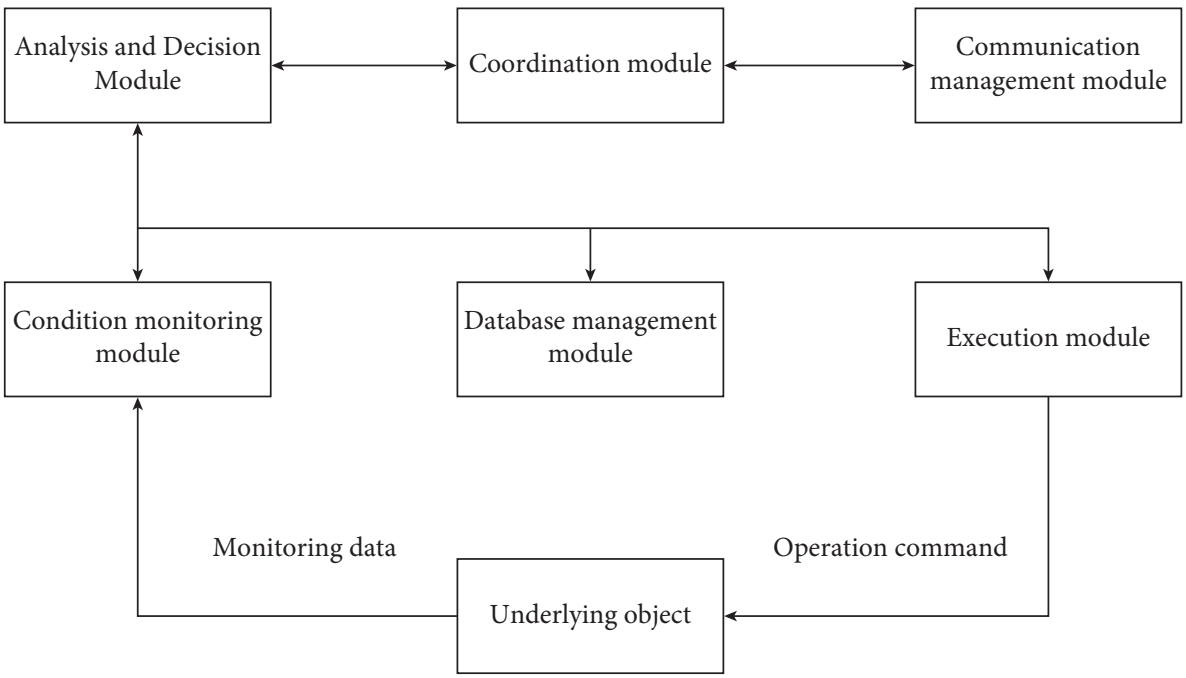

FIgURE 3: Design of a single agent.

subagent at the highest voltage level in the system, and $A_{i j}$ stands for the subsystem (substation) and equipment subagent at the subvoltage level in the system. The two-way connection line between the agents stands for the communication connection existing between the agents, and the solid line stands for the static connection (the connection has been fixed from the system startup to the shutdown and will not be released). The dotted line stands for dynamic connection (it is required to obtain the address of the other party from the server for information interaction, establish a two-way connection, and release the connection after the interaction to save the system resources).

In the implementation of the system, the communication information between the agents is formatted based on the unified FIPA standard. The interaction between the agents is reflected in two aspects: (1) Information sharing: the basic operating status and monitoring information of the power grid by the agents, as well as the analysis result information, can be acquired by other agents that have been granted authority. (2) Collaborate and coordinate to accomplish the tasks: the regulation and operation in the

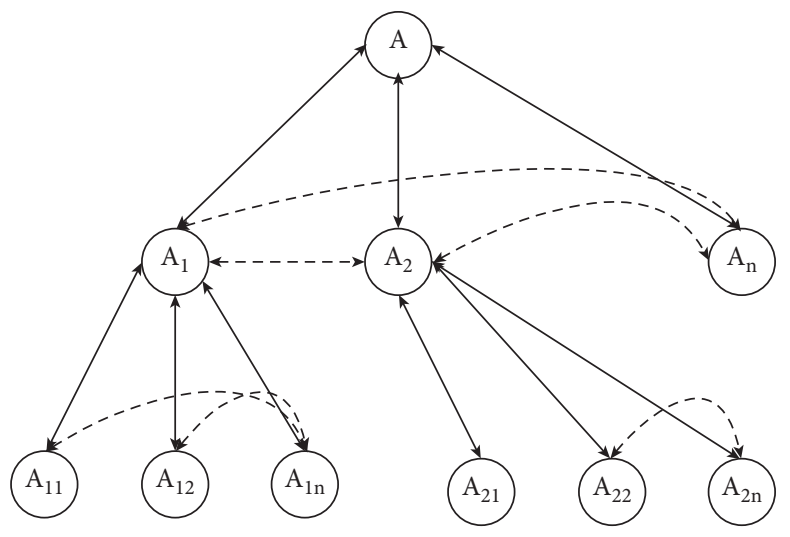

FIGURE 4: Design diagram of the multiagent system structure.

power grid are not independent. Instead, they are interrelated and mutually affected. Hence, the agents will send task assistance requests to the other agents based on the requirements of the tasks and request the operation of the automated electrical components or equipment of other 


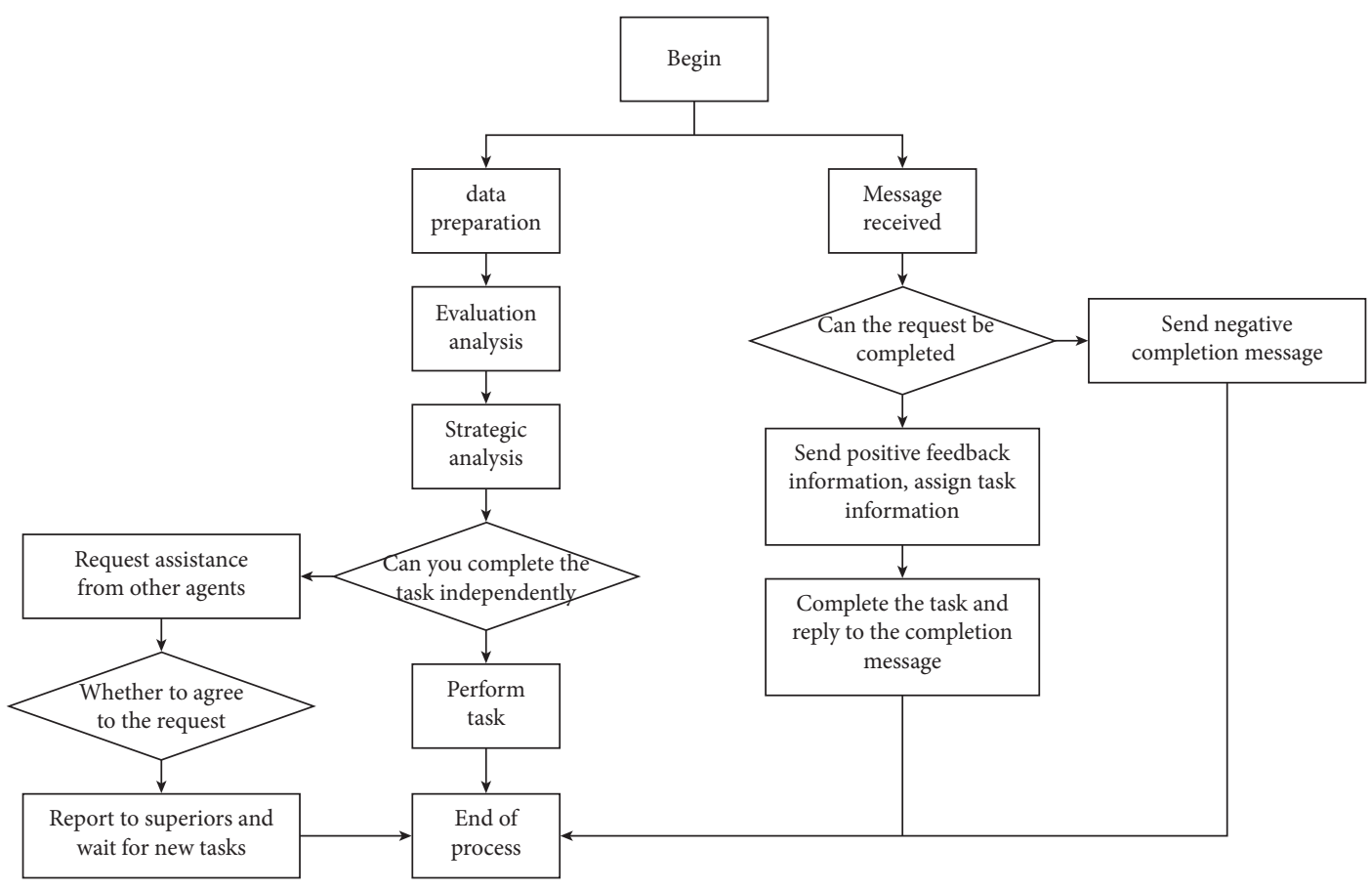

FIgURE 5: Workflow chart of a single agent.

agents. The other agents are required to determine and process the received requests and send back the processing results. The requester determines the actions in the next step based on the request processing results. The aforesaid two major types of information are subdivided into more specific information. Each type of information has a different tag. The analysis module will call different algorithms to process the information based on its tag.

\section{Simulation Implementation}

3.1. Establishment of the Multiagent System. The multiagent system is composed of single agents, and the specific workflow chart of the single agents is shown in Figure 5.

When the agent is idle, it is set to a standby state to reduce power consumption. As shown in Figure 5, there are two types of wake-up: active wake-up and passive wake-up:

(1) Active wake-up: the agent evaluates and regulates itself and the environment on a regular basis. The steps are described as follows:

(a) The agent actively sends measurement commands to each measurement unit at a regular interval, acquires the monitoring data from the database in real time, sends information sharing requests to other agents when necessary, and remotely obtains data from other agents.

(b) After the data preparation is completed, the agent analyzes and evaluates the system based on the data, stores the results in the work log, and reports the results to the superior agent. (c) With the evaluation results as the basis, the system conducts decision-making analysis and deploys the targeted strategic tasks.

(d) Determine whether the task can be completed independently. If the task is completed, the activity is recorded in the log and reported to the superior agent, and then the process is ended. If not, proceed to Step (e).

(e) Request assistance from the corresponding other agents to complete the task together. If a positive reply is obtained, proceed to Step (d) to complete the task to be completed by itself, and the process is ended. Otherwise, the situation is reported to the superior agent, waiting for the comprehensive control of the superior agent, and then the process is ended.

(2) Passive wake-up: in the standby state, the agent will receive information from the external environment. After it has received the requests from other agents, the system begins to analyze and evaluate the aforesaid requests, determine whether to execute the requests, and give the response accordingly. If it agrees to perform the task requested by the other party, it will wait for the specific task instructions from the other party after sending the message and reply to the other party regarding the completion status of the specific task after the task is completed. If the request is rejected, the process ends after the rejection message is sent, and the agent reenters the standby state, waiting for the next message. 
After the behavioral rules for system analysis, communication, collaboration, and other aspects of the single agent are designed, the operation mode of the multiagent system will be essentially determined as well.

After the agent is started, its first action is to initialize the communication module, send registration information to the communication management agent of the agent group that acts as a server, and register its related information, including name, location, category, working method, and communication IP address. After the registration is completed, the communication management agent stores the information of the registration agent in the communication database and adds the agent to the online agent list, which indicates that the agent is in a communicable state.

The multiagents have two communication modes, vertical and horizontal, as shown in Figure 6. In the horizontal communication mode, the address of the other party is obtained by sending an address request to the server, then a two-way connection for communication alone is established, and the link is released after the communication is completed. In the vertical communication mode, as the connection between the upper-level agent and the lower-level one has been maintained, messages can be sent directly to implement one-to-one or one-to-many communication with online agents. The accuracy and safety of information transmission are taken into consideration, and TCP/IP protocol is used for communication in normal circumstances. The connectionless UDP protocol communication is taken as a backup communication method for special moments.

The communication process between agents is described as follows:

(1) Action process of the agent of the sender:

(a) The analysis and decision-making module transmits the original information to be sent to the coordination module. The information shall include the information type label and the specific contents.

(b) The coordination module determines the priority of the message based on the type and content of the information and adds it to the information content.

(c) The communication module formats and packages the information according to the FIPA standards, adds attributes such as sender, time, and information ID, and places the original information into the content item (content). The protocol of the language used in the original information is encapsulated into the language item (language). After the encapsulation is completed, the communication module sends the information packet to the receiving agent according to the TCP/IP protocol.

(2) Action flow of the receiving agent:

(a) The communication module unpacks the received information, acquires the information attributes and contents, and transmits them to the coordination module.

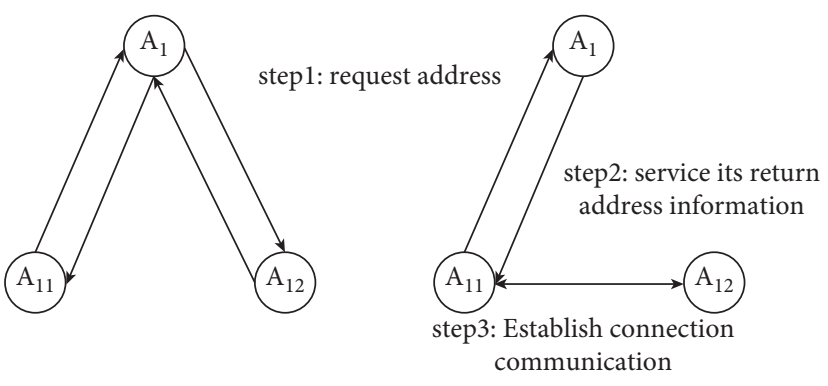

Vertical communication Horizontal communication

FIGURE 6: Schematic diagram of the communication mode.

(b) The coordination module consolidates the time, sender priority, and other indexes to determine the priority of the message, insert the information into the corresponding position of the message buffer area in the coordination module based on the determination result, and wait for processing.

(c) After the processing is completed, the analysis module starts to process the information. The analysis module calls the corresponding algorithm based on the type of message to carry out the analysis. If it is information sharing information, proceed to Step (d). If it is task request information, proceed to Step (e).

(d) Firstly, determine whether the reply message is sent by the system. If so, return to the process of sending the message in the analysis module and proceed to the next step. If not, call the information sharing information processing algorithm to analyze and process the information, acquire the information from the corresponding database or algorithm, and reply to the sender agent.

(e) Firstly, determine whether the reply message is sent by the system. If so, return to the process of sending the message in the analysis module and proceed to the next step. If not, call the task request information processing algorithm to analyze the information, and finally reply to the processing method of the aforesaid request to the sender agent.

3.2. Design of the Database. No. 1 main transformer of a substation is taken as an example to illustrate the specific working mode of the system, such as the temperature measurement and evaluation. The data include the temperature, electric field strength, the state of the cooling device, and the gas content in the oil as environmental measurement variables, as well as fixed data such as transformer parameters. For compliance with the comprehensive requirement of the algorithm, there is also some shared information from other devices; the measurement data are prestored in the database, and the actual situation is simulated for the reading sequence.

Based on the information that indicates the operating status of the transformer and the external environment acquired based on the wireless sensor network, the agent 
evaluates the health status of the transformer by calling the analysis algorithm in the analysis module. In this case, the evaluation result is that the transformer is in a seriously aging state, which is determined to have a high failure probability.

The agent can acquire the information on the evaluation results of other agents at the same level (transformer 2, transformer 3, and transmission line 2) as a reference, combined with the evaluation results of its own health status, and call the fault diagnosis and fault prediction algorithm to analyze the transformer. In this case, the primary consideration is that the transformer may have a relatively high probability of low energy discharge (44.8\%), low energy discharge and overheating (32.61\%), and other types of faults based on the results of DGA fault diagnosis according to the prediction. The corresponding decision and control strategy are made according to the analysis situation, and the analysis conclusion is drawn to perform electric isolation of the transformer. In addition, the maintenance personnel are warned to carry out overhaul and replacement accordingly.

After the execution module receives the task for electrical isolation of the transformer from the decision-making, it determines the specific operation plan based on the specific grid situation: disconnect the circuit breakers 3311, 3310, 3312,3501 , and 1101 that are connected to the transformer. Among them, circuit breaker 1101 is connected to the equipment-level agent in transmission line 2. The agent sends a message to request the cooperation of the transmission line agent to disconnect circuit breaker 1101. After the overall operation is completed, the execution result is fed back and recorded in the log.

The above analysis and control results are reported to the upper-level system agent. After the upper-level system agent receives the notification on the application for the maintenance of No. 1 transformer, it reschedules the grid operation mode when the transformer is disconnected. The load of main transformer 1 is transferred and shared by transformers 2 and 3 , and the specific operation is completed through the execution module. This case has briefly explained the functions of the smart grid information management system based on the multiagent technology.

(1) Management and coordination agent: it coordinates and manages the relationship between multiple agents in the system and develops a series of decision-making plans for the choice of dispatchers based on the auxiliary decision-making information acquired from the qualitative and quantitative analysis provided by other agents in the system using the corresponding decision-making methods and knowledge.

(2) System monitoring agent: it monitors the operation of multiple application systems and collects online and offline information of all systems, including SCADA data, electric energy data, operating parameters of secondary substation equipment, image monitoring signals, and dispatching management system information, and consolidates the data to the management coordination agent.
(3) System control agent: it breaks down the integrated control commands automatically issued by the grid dispatcher and the system, such as mode adjustment, accident handling, and transactions on the power market, into single operations or sequences of program operations (remote control, remote adjustment, protection switch-on and return, fixed value change, etc.), and communicates them to each application system so that the operation can be completed by each application system accordingly.

(4) Economic operation agent: in the routine operation, it can apply the corresponding models and algorithms (such as load forecasting, economic dispatch, economic unit combination, hydropower plan, exchange plan, fuel plan, and maintenance plan) based on the safety and stability constraints with the guidance of the operating rules and support the development of strategies for the system-wide economic operation. During the regular operation, it continuously provides information about the overall economic efficiency, network loss, and thermal efficiency of the system that occur and put forward suggestions and solutions on how to reduce the loss and improve the efficiency (Figure 7).

(5) Safety analysis agent: the corresponding models and algorithms are applied in real time (such as the calculation of power flow, state estimation, real-time network analysis, scheduling of safety constraint, analysis of voltage stability, and analysis of predictive failure) and in support of safety assessments of the system. During the regular operation of the system, it can issue an early alert of distress to inform the weaknesses of the system in real time. At the same time, it can also offer suggestions and provide strategies to improve safety and stability so that the power system can always maintain a normal operating state.

(6) Human-computer interaction agent: the interfaces of various application systems are integrated and unified to implement a centralized virtual console for regional power grid dispatchers. It has an intuitive interface and can present the operation status of the regional power grid and equipment in the form of various graphics, dashboards, tables, sounds, animations, and images. Dispatchers can configure various kinds of display styles based on their own habits.

(7) Simulation training agent: the environment of virtual training for dispatching integrated professional business is implemented. The aforesaid professions include dispatchers, monitors, mode personnel, and load personnel at various levels. The training content not only includes the simulation operation of the power grid but also has integrated the various systems used by the dispatching professionals in practice, including the dispatching management system, the image monitoring system, the power site 


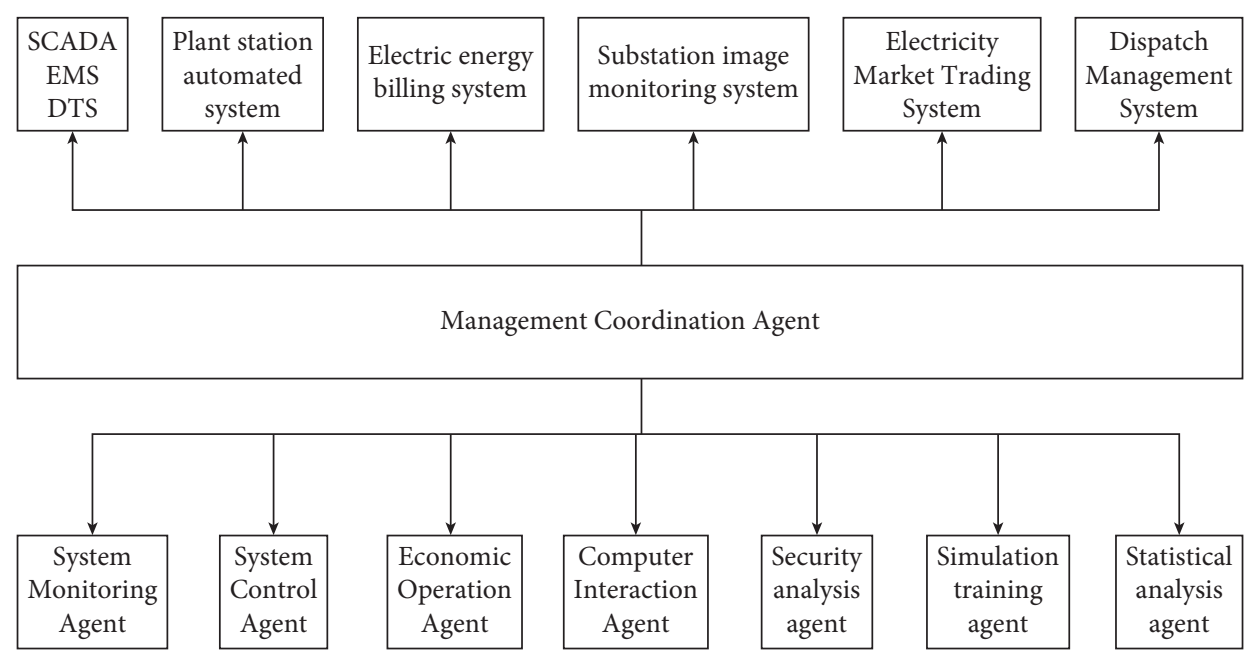

FIGURE 7: Architecture diagram of the intelligent dispatching system of a multiagent regional power grid.

management system, and the trading system of the power market. The training of functions is completed, such as power grid dispatching, filling of the operation ticket, load forecasting, method arrangement, power market transaction, handling of system accidents, and prediction of accidents.

(8) Electric power market agent: the scheme for power market operation is developed by collecting and consolidating information on grid operation, users, environment, policies, and other aspects, including the estimated load, planning, analysis, and statistics, to ensure that the regional power supply bureau can secure the most economic transactions in the electric power market.

(9) Statistical analysis agent: data information from various application systems is mined and extracted based on agent intelligence. The summary, analysis, statistics, classification, and other types of processing of data are completed according to different demands to form various reports and strategies to support decision-making. It has provided dispatchers with powerful data analysis tools, which can be used to prepare various reports flexibly and present the results of data analysis in multiple ways.

\section{Conclusions}

In this paper, the framework structure of an intelligent dispatching automation system based on the MAS technology is put forward, which can comply with the requirements of smart grid dispatching under the new situation. The intelligent dispatching automation system based on the MAS technology has more powerful functions than the existing EMS, with more scientific decision-making, which can provide a reliable guarantee for the regular operation of the power system. Based on the multiagent technology, an information management system with a hierarchical structure is established in this paper based on the features of multiagent collaboration for problem resolution. The strategy proposed has implemented the status monitoring, status evaluation, and status maintenance of the smart grid and provided technical support for manual maintenance.

\section{Data Availability}

The data used to support the findings of this study are available upon request to the author.

\section{Conflicts of Interest}

The author declares no conflicts of interest.

\section{References}

[1] N. Kumar, S. Zeadally, and S. C. Misra, "Mobile cloud networking for efficient energy management in smart grid cyberphysical systems," IEEE Wireless Communications, vol. 23, no. 5, pp. 100-108, 2016.

[2] M. M. Eissa, "First time real time incentive demand response program in smart grid with "i-Energy" management system with different resources," Applied Energy, vol. 212, no. 15, pp. 607-621, 2018.

[3] A. C.-F. Chan, J. Zhou, and A. Secure, "A secure, intelligent electric vehicle ecosystem for safe integration with the smart grid," IEEE Transactions on Intelligent Transportation Systems, vol. 16, no. 6, pp. 3367-3376, 2015.

[4] J.-S. Chou and N.-S. Truong, "Cloud forecasting system for monitoring and alerting of energy use by home appliances," Applied Energy, vol. 249, no. 1, pp. 166-177, 2019.

[5] X. Huang, J. K. Liu, S. Tang et al., "Cost-effective authentic and anonymous data sharing with forward safety," IEEE Transactions on Computers, vol. 64, no. 4, pp. 1-8, 2015.

[6] I. S. Bayram and T. S. Ustun, "A survey on behind the meter energy management systems in smart grid," Renewable and Sustainable Energy Reviews, vol. 3, no. 2, pp. 136-144, 2016.

[7] K. Alshehri, J. Liu, X. Chen, and T. Başar, "A game-theoretic framework for multiperiod-multicompany demand response management in the smart grid," IEEE Transactions on Control Systems Technology, vol. 2, no. 99, pp. 1-16, 2020. 
[8] A. Aktas, K. Erhan, S. Özdemir, and E. Özdemir, "Dynamic energy management for photovoltaic power system including hybrid energy storage in smart grid applications," Energy, vol. 162 , no. 1 , pp. $72-82,2018$.

[9] A. Ghasemi, H. Shayeghi, M. Moradzadeh, and M. Nooshyar, "A novel hybrid algorithm for electricity price and load forecasting in smart grids with demand-side management," Applied Energy, vol. 177, no. 2, pp. 40-59, 2016.

[10] Q. Wu and M. Wu, "SU-E-T-241: design and implement of an information management system for radiation workers in a hospital," Medical Physics, vol. 42, no. 6, pp. 3388-3394, 2015.

[11] N. Takayanagi and N. Matsuda, "Information processing system, data management method, information processing apparatus, and control method and control program therefor," Energy Sources, Part A: Recovery, Utilization, and Environmental Effects, vol. 36, no. 10, pp. 1134-1140, 2017.

[12] S. T. Hamman, K. M. Hopkinson, and J. E. Fadul, "A model checking approach to testing the reliability of smart grid protection systems," IEEE Transactions on Power Delivery, vol. 32, no. 6, pp. 2408-2415, 2017.

[13] F. Luo, G. Ranzi, W. Kong, Z. Y. Dong, S. Wang, and J. Zhao, "Non-intrusive energy saving appliance recommender system for smart grid residential users," IET Generation, Transmission \& Distribution, vol. 11, no. 7, pp. 1786-1793, 2017.

[14] W. Tushar, C. Yuen, B. Chai et al., "Smart grid testbed for demand focused energy management in end user environments," IEEE Wireless Communications, vol. 23, no. 6, pp. 70-80, 2016.

[15] J.-S. Chou and N.-T. Ngo, "Time series analytics using sliding window metaheuristic optimization-based machine learning system for identifying building energy consumption patterns," Applied Energy, vol. 177, no. 11, pp. 751-770, 2016. 